\title{
Indications and Therapeutic Potential of Virtual Reality in Pediatric Dentistry
}

\author{
Do $\mathrm{NM}^{1}$, Graff $\mathrm{D}^{2}$, Chauvin $\mathrm{C}^{2,3}$, Manière $\mathrm{MC}^{4}$ and Clauss $\mathrm{F}^{1 *}$ \\ ${ }^{1}$ Doctor of Dental Surgery, University of Strasbourg, University Hospital of Strasbourg (HUS), Department of Pediatric Dentistry and \\ National Reference Center for Oro-dental manifestations of rare diseases ORARES, France
}

${ }^{2}$ Doctor of Medicine, Anesthesiologist in Private Practice, Department of Pediatric Anesthesia and Critical Care, University Hospital of Strasbourg (HUS), France

${ }^{3}$ University Hospital of Strasbourg (HUS), Department of Pediatric Anesthesia and Critical Care, France

${ }^{4}$ Doctor of Dental Surgery, Full Professor in the Department of Pediatric Dentistry and National Reference Center for Oro-dental manifestations of rare diseases ORARES, University of Strasbourg, University Hospital of Strasbourg (HUS), France

*Corresponding author: François Clauss, Full Professor of Pediatric Dentistry, University of Strasbourg, University Hospital of Strasbourg (HUS), Department of Pediatric Dentistry and National Reference Center for Oro-dental manifestations of rare diseases orares, France

\begin{abstract}
In the age of technology, Virtual Reality (VR) has stormed into many aspects of our lives, the medical field is no exception. According to Global Industry Analysts, the VR market in medicine is expected to grow to $\$ 2,2$ billion by 2027 . This technology hold promises in revolutionizing the healthcare industry. Its applications are ranging from training medical professionals (with possibilities for students to better assess medical notions or to treat virtual patients during their pre-clinical course) to diagnosing and treating various conditions. The user is immerged into a tri-dimensional multi-sensory ambiance and his focus is solely on the scenario. It's an immensely powerful tool for probing the relationship between emotion and audio/visual perception. As imaginative and inquisitive as children are, the immersive quality of VR is most likely to amaze them. Their attraction for new technologies combined with their ease to escape to a specific scenario through movies or videogames can make the VR experience, and therefore the medical or dental procedure, more pleasant for them. This article summarizes the therapeutic potential of VR in pediatric dentistry through its anxiolytic and analgesic effects.
\end{abstract}

Keywords: Virtual reality; Analgesia; Anxiolytic; Distraction; Dental; Children

\section{Introduction}

The coronavirus pandemic and its social restrictions have helped boost our interest in Virtual Reality (VR) when it comes to entertainment and business, fields for which it was originally designed for. Its applications also expand to the healthcare field [1], especially in pediatric dentistry [2].

\section{Use of virtual reality in pediatric dentistry}

VR is a computer technology that creates an artificial 3-dimensional simulated environment through audio and visual immersion. It consists of a head mounted display and a pair of goggles that are connected to either a computer or a cell phone.
The headset has sensors that track user's head movements, creating the illusion of moving around in the virtual space [3]. The VR scenario needs to be adapted to the context of dental treatment, with calm and anxiolytic scenarios, avoiding any agitation, hyperstimulation, or head movements during the procedures. However, the child is much more than a simple passive recipient of VR: he becomes an active collaborator of his treatment through the choice of the virtual reality environment. While in VR, he can interact with virtual characters controlled by computer algorithms called embodied agents. The first-person view in VR creates the illusion of being surrounded by the virtual environment and sharing the same 
physical space with the embodied agent [3]. In addition, VR can create realistic simulations by allowing users to interact with the virtual environment using naturalistic movement, similar to how they would navigate the physical world. Research with children has shown that the brain can respond to VR stimuli as if it were real [3]. Anxiety is often linked to pain and VR was found effective, not only on anxiety, but also in pain relieve.

When it comes to pediatric dentistry, as the patient's attention is focused on the VR scenario, the visual and audio stimuli that usually trigger anxiety are shunted. The reduction in fear in pediatric needle procedure is significant while using VR compared with standard methods of behavioral management or distraction [4]. Two recent meta-analysis, published in 2019 and 2020, have investigated the physiological response in children who received virtual reality devices during dental procedures. The first one concluded that children with VR have a significant lower heart rate during local dental anesthesia (MD, -3.78; 95\% CI, -6.73, -0.83; $\mathrm{p}=0.01$ ). This result is based on 352 participants across 6 studies. However, there was no significant change for the oxygen saturation level [5]. Unlike the first meta-analysis, the second shows no significant improvement linked to VR use during the administration of local anesthetic and the placement of the surgical site. They concluded, however, that VR audiovisual distraction may reduce pain perception and improve child behavior during decay removal and restoration placement [6]. VR is also indicated for cognitive and behavioral therapies based on progressive exposition to different stimuli such as the dental anesthesia needle. Psychological preparation for a surgical intervention by virtual visit of the operating room is possible and allows a reduction in preoperative anxiety scores [7]. The main objective and interest of VR, in these cases, is the cognitive reprogramming of anxious children. Furthermore, there is growing interest in nonpharmacological techniques for anxiety and pain management. Patients who are intolerant to pharmalogical conscious sedations such as inhalation with nitrous oxide and oxygen or who cannot benefit from an administration of benzodiazepines (midazolam) may also be treated under virtual reality. However, given the nature of the procedure, the contraindications to the use of virtual reality mainly refers to certain psychopathological contexts (claustrophobia), a history of post-operative nausea and vomiting, growth retardation or young age leading to an unsuitability of the virtual reality mask. Another contraindication to the use of virtual reality is susceptibility to motion sickness.

\section{Medical hypnosis in pediatric dentistry}

One of the main non-pharmacological distraction therapies used in pediatric dentistry is medical hypnosis [8]. In children, clinical hypnotherapy techniques including hypoalgesia have relieved acute pain associated with local anesthesia and therefore help allay fear or increase patient cooperation [9]. According to the American Psychological Association, medical hypnosis induces a "state of modified consciousness involving focused attention and reduced peripheral awareness characterized by an enhanced capacity for response to suggestion". Milton Erickson developed a permissive, accommodating, and indirect approach, often called Covert or Conversational Hypnosis. This technique is particularly interesting for children, who have a natural talent for playing and who find it easy to enter into an imaginary world. Three fundamental conditions remain essential in hypnosis: patient's motivation, patient's cooperation, and patient's confidence in the therapist [10]. One study demonstrated that hypnosis was effective in reducing preoperative anxiety in the group of children receiving local dental anesthesia [11]. The mean modified Yale Preoperative Anxiety Score was 50\% lower in the Hypnosis group than in the Non-Hypnosis group at the time of anesthesia [11]. There were less crying and decreased pulse rate [11]. Hypnosis also modified the pain tolerance threshold, as shown by the Visual Analogue Scale (VAS) scores, with $86 \%$ of children from the Hypnosis group compared to $40 \%$ from the Non-Hypnosis group having a VAS score under 3 [11]. Another study found that children under hypnosis exhibited significantly less resistance to administration of local anesthesia $(\mathrm{P}<0.05)$ and there also was a significant difference in pulse rate, attributable to the hypnotic condition $(\mathrm{P}=.000)$, but not in oxygen saturation level [9].

A recent Cochrane systematic review attempted to answer the question: What is the effectiveness of hypnosis (with or without sedation) for behavior management of children who are receiving dental care in order to allow successful completion of treatment? Although there are many case reports indicating the benefits of hypnosis in pediatric dentistry, only 3 met the inclusion criteria and the authors concluded that further controlled trials were necessary to suggest the beneficial effects of hypnosis [12]. Given well-known developmental and inter-individual variations in suggestibility, researchers may want to confine studies to limited age ranges or to include sufficient numbers of children from various age groups to allow analyses of adequate statistical power within age categories. Besides, the main difficulties for comparing medical hypnosis between clinical studies correspond mainly to the inherent heterogeneity of the hypnosis speech. The variability of the hypnosis speech between practitioners makes it hard to compare them.

\section{Medical hypnosis through virtual reality}

Several recent software offering a multisensory immersion in sync with a hypnosis suggestion speech allow a more standardized hypnosis therapy. It combines the benefits of the visual distraction through VR with synopsis scripts based on cardiac coherence and suggestion. It often includes musical constructions that have been clinically proven to affect the patient's heart rate through relaxation, associating the ambient sounds with music therapy compositions. 
Hypnogram $@$ is one that software that are easy and clinically ready-to-use and don't require hypnosis skills for the practitioner. The patient can choose between different VR scenarios, voice and music atmosphere. It's a class 1 medical device, proven effective as a natural anxiolytic. It can be used as replacement of premedication with a long-lasting non-pharmacological anxiolytic effect. In association with local or regional anesthetic adjunct, or during the induction for general anesthesia, it provides analgesia with reduced side effects. Post-operative hypoalgesia can reduce pain and opioid use with a faster recovery. When it comes to pediatric dentistry, our pilot clinical study provides preliminary evidence of the feasibility of using immersive, interactive VR to distract pediatric dental patients and increase cooperation and of children during dental procedures. 12 patients were included, and the main inclusion criteria corresponded to an age comprised between 9 and 13 years old, without any neurological or psychiatric disease, in absence of previous post-operative vomiting or nausea. Our preliminary results show a better tolerance and global experience of dental procedure in a large range of procedures from oral surgery to endodontics or conservative care.

Long clinical sessions (lasting more than 1 hour) were also carried out under virtual reality, as part of complex rehabilitations of dental developmental anomalies, such as Amelogenesis Imperfecta or Molar Incisor Hypomineralisation, with a good level of cooperation from the patient. When used in conjunction with local anesthesia, VR procedure decreases anxiety and reduces pain and the need for sedatives and side effects, with perioperative anxiety score $45 \%$ lower and a satisfaction rate of $8 / 10$. The next step in our clinical research is a prospective controlled clinical study aiming to compare this VR device with the nitrous oxide pharmacological sedation in the context of anxious children undergoing conventional dental treatment. Our main experimental objective is to investigate the effectiveness of VR on anxiety and pain, as well as to compare it with the standard option of care for anxious children corresponding to inhalation conscious sedation with nitrous oxide and oxygen. Success and prognosis factors such as child's temperament will be discussed as they may influence suggestibility in children and overall efficacy of VR procedure.

\section{Conclusion}

Recent mass production of immersive VR goggles has increased their availability and affordability, and there is growing interest in non-pharmacological techniques for pain management, making VR analgesia a promising direction for future clinical research. Associating with medical hypnosis scenario, it combines the benefits of both distraction through VR and hypnosis. Children can discover the dental office in a playful and fun way.

\section{References}

1. Al Harasi S, Ashley PF, Moles DR, Parekh S, Walters V (2017) Hypnosis for children undergoing dental treatment. The Cochrane Database of Systematic Reviews 1(6).

2. Anthonappa RP, Ashley PF, Bonetti DL, Lombardo G, Riley P (2017) Nonpharmacological interventions for managing dental anxiety in children. The Cochrane Database of Systematic Reviews.

3. Atzori B, Lauro Grotto R, Giugni A, Calabrò M, Alhalabi W, et al. (2018) Virtual Reality Analgesia for Pediatric Dental Patients. Front Psychol 9: 2265.

4. Bohil CJ, Alicea B, Biocca FA (2011) Virtual reality in neuroscience research and therapy. Nature Reviews Neuroscience 12(12): 752-762.

5. Custódio NB, Costa F Dos S, Cademartori MG, Da Costa VPP, Goettems ML (2020) Effectiveness of Virtual Reality Glasses as a Distraction for Children During Dental Care. Pediatr Dent 42(2):93-102.

6. Erickson MH (2009) Naturalistic techniques of hypnosis. 1958. Am J Clin Hypn 51(4):333-340.

7. Huet A, Lucas Polomeni MM, Robert JC, Sixou JL, Wodey E (2011) Hypnosis and dental anesthesia in children : A prospective controlled Study. Int J Clin Exp Hypn 59:424-440.

8. Indovina P, Barone D, Gallo L, Chirico A, De Pietr, G, et al. (2018) Virtual Reality as a Distraction Intervention to Relieve Pain and Distress During Medical Procedures: A Comprehensive Literature Review. Clin J Pain 34(9):858-877.

9. Oberoi J, Panda A, Garg I (2016) Effect of Hypnosis during Administration of Local Anesthesia in Six- to 16-year-old Children. Pediatr Dent 38(2):112-115.

10. Ryu JH, Park SJ, Park JW, Kim JW, Yoo HJ, et al. (2017) Randomized clinical trial of immersive virtual reality tour of the operating theatre in children before anaesthesia : Virtual reality tour and preoperative anxiety. British Journal of Surgery 104(12):1628-1633.

11. Shetty V, Suresh LR, Hegde AM (2019) Effect of Virtual Reality Distraction on Pain and Anxiety During Dental Treatment in 5 to 8 Year Old Children. J Clin Pediatr Dent 43(2):97-102.

12. Zhang C, Qin D, Shen L, Ji P, Wang J (2019) Does audiovisual distraction reduce dental anxiety in children under local anesthesia? A systematic review and meta-analysis. Oral Dis 25(2):416-424. 
This work is licensed under Creative Commons Attribution 4.0 License

To Submit Your Article Click Here: Submit Article

DOI: $10.32474 /$ SJPBS.2020.04.000192

\begin{tabular}{|c|c|}
\hline SJPBS & $\begin{array}{c}\text { Scholarly Journal of Psychology } \\
\text { and Behavioral Sciences }\end{array}$ \\
\hline & $\begin{array}{l}\text { Assets of Publishing with us } \\
\text { - Global archiving of articles } \\
\text { - Immediate, unrestricted online access } \\
\text { - Rigorous Peer Review Process }\end{array}$ \\
\hline $\begin{array}{l}\text { Scholarly Journal of } \\
\text { sychology \& Behavioral sciences }\end{array}$ & $\begin{array}{l}\text { - Authors Retain Copyrights } \\
\text { - Unique DOI for all articles }\end{array}$ \\
\hline
\end{tabular}

